\title{
The Complicated Uncomplicated Epidural Placed Under General Anesthesia: A Complete Spinal in the Post- Anesthesia Recovery Unit
}

\author{
Jason Bryant ${ }^{\mathrm{a}, \mathrm{b}, \mathrm{c}}$, Anita Joselyn ${ }^{\mathrm{a}}$, Joseph Tobias ${ }^{\mathrm{a}, \mathrm{b}}$
}

\begin{abstract}
Placement of an epidural catheter under general anesthesia in children is associated with more neurologic complications as compared to placement when the patient is awake. The anesthetized patient cannot communicate the symptoms of an intrathecal placement. A 5-year-old boy had an epidural catheter placed after induction of general anesthesia. Negative aspiration of cerebral spinal fluid (CSF) was confirmed. The patient had intraoperative hypotension without bradycardia during the use of the catheter. In the post-operative recovery unit, the patient developed apnea and signs of a high spinal. Intrathecal placement of an epidural catheter is not a rare or unexpected complication of epidural analgesia. However, in an anesthetized child with negative aspiration of CSF combined with the inability of the patient to communicate the signs of a high spinal a delay in diagnosis may occur. Our case report highlights the importance of identifying intrathecal placement of an epidural catheter under general anesthesia.
\end{abstract}

Keywords: Epidural; Intrathecal; Subarachnoid; Complication; Pediatric anesthesia

\section{Introduction}

In the adult population, epidural catheters are placed prior to the induction of anesthesia given the perceived risk of an increased incidence of neurologic complications as compared to placement in an anesthetized patient [1]. Epidurals in pediatrics are often placed under general anesthesia given their cognitive level and inability to cooperate with the procedure $[2,3]$. A recent review of the pediatric regional anesthesia network

Manuscript accepted for publication September 08, 2014

aDepartment of Anesthesiology \& Pain Medicine, Nationwide Children's Hospital, Columbus, OH, USA

bepartment of Anesthesiology, Ohio State University, Columbus, OH, USA ${ }^{\mathrm{c} C o r r e s p o n d i n g ~ A u t h o r: ~ J a s o n ~ B r y a n t, ~ D e p a r t m e n t ~ o f ~ A n e s t h e s i o l o g y ~ \& ~ P a i n ~}$ Medicine, Nationwide Children's Hospital, 700 Children's Drive, Columbus, OH 43205, USA. Email: jason.bryant@nationwidechildrens.org

doi: http://dx.doi.org/10.14740/jmc1938w
(PRAN) indicated that $95 \%$ of pediatric regional anesthesia blocks are placed after the induction of general anesthesia [4].

We present a 5-year-old child who had an epidural placed for postoperative analgesia. Despite the lack of cerebral spinal fluid (CSF) return from the catheter, both during placement and subsequent use, total spinal blockade occurred. Also the lack of changes in heart rate is important as this may differ in the pediatric patient from the adult. The importance of prevention, early detection and treatment of complications following an inadvertent intrathecal placement of an epidural catheter are discussed. Formal consent for publication was obtained from the patient's guardian.

\section{Case Report}

A $17 \mathrm{~kg}$ 5-year-old boy with a past medical history of a urogenital cyst presented for surgical removal. Consent was obtained for a combined epidural-general anesthesia from the parents. The patient underwent an uneventful inhalational induction and intubation. His baseline pre-operation vitals included a blood pressure of 101/64 and a heart rate of 84 . His vital parameters were stable during induction. With the patient in the right lateral position, an epidural catheter was placed at T 10-11 space. Epidural space was identified at $1.5 \mathrm{~cm}$ depth by loss of resistance technique without imaging or ultrasound. The epidural catheter was advanced without resistance. There was negative aspiration for blood and CSF. The test dose was negative for a change in heart rate (however, with the patient under general anesthesia motor or sensory levels could not be assessed). Once the child was placed supine for surgery, the epidural catheter was dosed with $4 \mathrm{~mL}$ of $0.5 \%$ ropivacaine and a second dose of $4 \mathrm{~mL}$ was given $10 \mathrm{~min}$ later prior to incision. The blood pressure decreased to 52/31 after dosing but the heart rate remained stable. A fluid bolus was given, and the inhaled anesthetics were decreased which resulted in an improvement of the blood pressure to $80-90 \mathrm{~mm} \mathrm{Hg}$ systolic. The patient did not receive further doses of local anesthetic during the course of the 3-h surgery. The patient's trachea was extubated immediately following the surgical procedure when he was awake. In the post-anesthesia care unit (PACU), the patient complained of pain, rating it as a $8 / 10$ on a visual analog scale. At this time, the interval between the last doses of ropivacaine was $3 \mathrm{~h}$. The patient was given $2 \mathrm{~mL}$ of $0.5 \%$ 
ropivacaine after negative aspiration through the indwelling catheter. Within $5 \mathrm{~min}$, the patient was somnolent, complaining of ascending bilateral numbness and had diminished tidal volumes but responsive. After $7 \mathrm{~min}$, the patient was unresponsive, apneic and required bag mask ventilation. No change in heart rate was noted although there was a decrease in blood pressure from $98 / 79$ to $79 / 42$ which was treated with a fluid bolus. During this event, there was no change in the heart rate and no ECG abnormalities were noted. Pupil examination revealed bilateral mydriasis. Oxygen saturations were maintained above $94 \%$ during this period. The patient remained apneic for $20 \mathrm{~min}$. After $20 \mathrm{~min}$, spontaneous respirations returned. There was a fast regression of analgesic levels and the patient complained of pain after $50 \mathrm{~min}$. At this time, the epidural catheter was checked again for CSF or blood which was negative. The "epidural" catheter was then started on an infusion of $0.2 \%$ ropivacaine with $2 \mu \mathrm{g} / \mathrm{mL}$ of fentanyl at a rate of $1 \mathrm{~mL} / \mathrm{h}$. This infusion produced an analgesic level of c6 within 15 min while sitting up right. The drip was immediately discontinued and the catheter was removed. Upon reflection, this may have not been the optimal choice in management and intrathecal placement without the ability to aspirate CSF could have been suspected sooner. Upon removal of the catheter, intermittent aspiration was attempted. As the catheter was pulled out $1 \mathrm{~cm}, 3 \mathrm{~mL}$ of easy flowing clear fluid was obtained. Patient-controlled analgesia with morphine was started in the PACU. The patient regained full motor and sensory function prior to leaving the recovery room. Follow-up revealed that he had a non-positional headache at day 2 that resolved without any further interventions and he was discharged home without any residual neurological deficits.

\section{Discussion}

Our case illustrates the importance of identifying the unintentional intrathecal placement of what appeared to be an "uncomplicated" epidural catheter placed under general anesthesia. The placement of the epidural catheter in our patient was uneventful following negative aspiration for blood and CSF. The lack of CSF from the needle during epidural placement may have been secondary to the catheter puncturing the dura during advancement. The development of profound motor and sensory blockade following the test dose could not be assessed given that this was performed in an anesthetized child. The $50 \%$ decrease in systolic blood pressure that occurred following the initial loading dose should be interpreted as the initial warning sign. Although such changes can occur with the sympathetic blockade achieved with epidural analgesia especially in the setting of mild perioperative hypovolemia a significant decrease in blood pressure is a warning sign of possible intrathecal placement.

A subdural or intradural placement was considered in this case but with the fast onset, complete (not "patchy") bilateral block and low volume of anesthetic, it does not fit the clinical features [5]. There also have been cases of intradural catheters migrating or leaking into the intrathecal space causing total spinal blocks confirmed on imaging which may explain the lack of the ability to aspirate CSF [6]. These cases still presented with a slow patchy block that progressed to a total spinal blockade.

The analysis of the PRAN database revealed very low incidence of complications following central neuraxial block in children [4]. Although rare, there are reports of serious neurological complications following epidural block in children as shown by the United Kingdom Epidural Audit [7]. Another anecdotal series includes not only permanent neurologic sequelae but also a patient whose presentation was similar to our case [8]. This case was an 11-year-old boy who had an uneventful thoracic level epidural placed under general anesthesia. There was significant hypotension following the test dose and loading dose with no changes in the heart rate. An epidural bolus in the PACU was followed by hypotension and apnea requiring bag and mask ventilation. Removal of the epidural catheter was followed by leakage of a small amount of CSF at catheter insertion site. This case report similar to ours highlights the possibility of having an intrathecal placement of epidural catheter in children under general anesthesia despite negative aspiration for CSF. The significant hypotension (greater than $20 \%$ ) following test dose and loading dose in both the instances provides the clue that the epidural catheter was intrathecal. The lack of changes in heart rate was similar and questions the assumption that a high thoracic level block will usually result in bradycardia as is seen in the adult population.

Provisional recommendations for epidural anesthesia in anesthetized children have been provided by Berde and Greco including the statement "Severe hypotension following test dose can be assumed to be due to subarachnoid placement unless demonstrated otherwise" [2]. The definition of severe hypotension is open for interpretation. The $50 \%$ decrease with the initial bolus is easily defined as significant. However, with the second bolus in the PACU, the decrease was less at $19.4 \%$ but was associated with clinical signs of intrathecal injection since the patient was awake at the time. Fortunately the hypotension was treated and no long-term sequelae were noted in our patient. However, the earlier recognition of an intrathecal catheter would have led to closer management in the PACU and would have prevented the life-threatening apnea. If the patient had been located in a less acute environment when he received his ropivacaine bolus such as the surgical hospital floor, a delay may have occurred in the ability to ventilate the patient the outcome that may not have been favorable.

The intrathecal placement may not always be associated with CSF aspiration or change in heart rate as highlighted in our case report. Given placement during general anesthesia, a profound motor and sensory response to the test dose, one of the primary signs of unintentional intrathecal injection will not be noted. However, significant hypotension with or without bradycardia following a test dose or a loading dose should alert the anesthesiologist about the possibility of epidural catheter being within the intrathecal space.

\section{References}

1. Horlocker TT, Wedel DJ. Neurologic complications of 
spinal and epidural anesthesia. Reg Anesth Pain Med. 2000;25(1):83-98.

2. Berde C, Greco C. Pediatric regional anesthesia: drawing inferences on safety from prospective registries and case reports. Anesth Analg. 2012;115(6):1259-1262.

3. Krane EJ, Dalens BJ, Murat I, Murrell D. The safety of epidurals placed during general anesthesia. Reg Anesth Pain Med. 1998;23(5):433-438.

4. Polaner DM, Taenzer AH, Walker BJ, Bosenberg A, Krane EJ, Suresh S, Wolf C, et al. Pediatric Regional Anesthesia Network (PRAN): a multi-institutional study of the use and incidence of complications of pediatric regional anesthesia. Anesth Analg. 2012;115(6):1353-1364.
5. Collier CB. Accidental subdural injection during attempted lumbar epidural block may present as a failed or inadequate block: radiographic evidence. Reg Anesth Pain Med. 2004;29(1):45-51.

6. Collier CB. The intradural space: the fourth place to go astray during epidural block. Int J Obstet Anesth. 2010;19(2):133-141.

7. Llewellyn N, Moriarty A. The national pediatric epidural audit. Paediatr Anaesth. 2007;17(6):520-533.

8. Meyer MJ, Krane EJ, Goldschneider KR, Klein NJ. Case report: neurological complications associated with epidural analgesia in children: a report of 4 cases of ambiguous etiologies. Anesth Analg. 2012;115(6):1365-1370. 